\title{
Evaluación de la influencia de variables socioeconómicas en la práctica del running en Argentina
}

\author{
Evaluation of the Influence of Socioeconomic Variables on the \\ Running Practice in Argentina
}

\author{
Ronald Uthurralt \\ Universidad Católica Argentina, Buenos Aires, Argentina \\ Doctorando en Sociología, Universidad Católica Argentina
}

\begin{abstract}
RESUMO: 0 objetivo deste trabalho é analisar a relação entre diferentes variáveis socioeconômicas (idade, sexo, escolaridade, moradia, renda, ocupação e hábitos esportivos) com a prática do running na Argentina. Adotamos uma abordagem quantitativa, com base na Pesquisa Nacional de Fatores de Risco (ENFR) 2009. Estabelecemos categorias de acordo com o grau de conexão com o mundo social da corrida e analisamos a conexão de diferentes variáveis socioeconômicas com as categorias de análise. Para isso utilizamos regressões logísticas e análise de correspondências múltiplas. Nossas análises mostram que as características individuais, sociais e econômicas têm um efeito relevante sobre a participação no running. Em geral, os corredores tendem a ser homens de meia-idade, renda médiaalta e ensino superior.
\end{abstract}

PALAVRAS-CHAVE: Running; análise quantitativa; participação; variáveis socioeconômicas.

ABSTRACT: The aim of this study is to analyze the relationship among socioeconomic variables (age, gender, income, occupation and sport habits) with the running practice in Argentina. A quantitative approach was adopted, based on the National Survey of Risk Factors (ENFR) 2009. We established categories based on their relationship with the running social world and we analyzed the relationship among different socioeconomic variables with our research categories. Logistic regressions and multiple correspondence analysis we used along this work. Our analyses show that individual, social and economic characteristics have a relevant effect on running participation. In general terms, runners tend to be males, middle aged, with middle high income and higher education.

KEYwordS: Running; Quantitative Analysis; Participation; Socioeconomic Variables. 


\section{INTRODUCCIÓN}

El running es una actividad de masas, con millones de corredores aficionados en todo el mundo, pero originalmente era una actividad deportiva para atletas competitivos. Hasta fines de la década del 60, el correr en la calle o en parques era una actividad extraña, si la gente corría en público se debía a que estaba apurada. Los primeros corredores se avergonzaban de correr en público y la situación era aún peor en el caso de las mujeres. Correr entre peatones como forma de ocio no se correspondía con los códigos sociales vigentes. ${ }^{1}$ A partir de entonces, el desarrollo de una mayor informalidad en las relaciones sociales facilitó que las personas realizaran actividades físicas en público, mientras que el proceso de difusión de la actividad hizo que el running, inicialmente en Estados Unidos y luego en Europa, pasara de ser una actividad competitiva limitada a un grupo pequeño de deportistas especializados a convertirse en una actividad recreativa de masas. A partir de los años 90, en lo que Scheerder, Breedveld y Borgers denominan la "segunda ola del running", la actividad alcanzó una nueva etapa de crecimiento, transformándose en un fenómeno global. El crecimiento del running en Argentina forma parte de esta segunda ola. Desde fines de la década del 90 el furor por correr aumenta año tras año, reflejado no sólo en la marea de corredores que copan los espacios públicos, sino también en la creciente cantidad de competencias e inscriptos. ${ }^{2}$

El crecimiento de la epidemia de obesidad y el estancamiento en la adopción de prácticas deportivas en los países desarrollados durante la primera década de este siglo trajeron aparejados un creciente interés académico por el estudio de los determinantes de la participación deportiva, tanto desde lo teórico como lo empírico. Los estudios empíricos de participación deportiva, anteriores al auge del running, comenzaron a realizarse en Estados Unidos a finales de la década de 1960. Inicialmente se basaron en modelos lineales de cuadrados mínimos y evolucionaron

\footnotetext{
${ }^{1}$ SCHEERDER; BREEDVELD; BORGERS. Running across Europe: the rise and size of one of the largest sport markets, p. 1.

${ }^{2}$ HIJÓS. La historia del running en Argentina, p. 122. La pandemia de Covid-19 representó una seria disrupción para la actividad. La práctica deportiva en espacios públicos estuvo prohibida o seriamente restringida durante varios meses de 2020.
} 
posteriormente a modelos logísticos de diferente nivel de complejidad. Downward ${ }^{3}$ desarrolló un modelo de participación deportiva de acuerdo con un enfoque que distingue características individuales y sociales, variables económicas y características deportivas. Para ello desarrolló, a partir de los datos de la General Household Survey 2002 del Reino Unido, un modelo de regresión logística, con la participación deportiva como variable dependiente dicotómica y diversas características o factores como variables independientes, que tomamos como guía.

García, Lera-López y Suárez ${ }^{4}$ sostienen que, a pesar de las dificultades para comparar entre los estudios -debidas a las diferencias en las actividades consideradas y en las formas de medición- es posible hacer una evaluación general de la influencia de ciertas variables económicas, individuales y sociales. La evidencia empírica muestra que la probabilidad de participación deportiva disminuye con la edad, lo que puede atribuirse a limitaciones físicas y biológicas. El género también es un aspecto que influye significativamente en la probabilidad de participación ya que, en general, los hombres participan en mayor medida que las mujeres en actividades deportivas y lo hacen con una frecuencia mayor. Estas diferencias podrían atribuirse a factores biológicos, culturales y sociales, vinculados a diferencias tanto en las responsabilidades familiares como en lo relativo al comportamiento, las expectativas sociales y el trabajo. Los estudios empíricos brindan evidencia de que los bajos ingresos pueden actuar como una barrera para la práctica deportiva, aunque parecen no influir en la frecuencia de dicha práctica. Las restricciones de tiempo, medidas a partir de la ocupación y calificación profesional, el tamaño del hogar y el estado civil, aparecen también como un factor limitante. Ciertas ocupaciones de los segmentos socioeconómicos más bajos se asocian negativamente con la práctica deportiva. Lo mismo sucede con hogares grandes y con las personas casadas. Mayores niveles de educación se asocian a una mayor probabilidad de participación deportiva, posiblemente asociada a una mayor conciencia de los beneficios del deporte y a unos mayores ingresos por hora trabajada.

\footnotetext{
${ }^{3}$ DOWNWARD. Exploring the economic choice to participate in sport: Results from the 2002 General Household Survey, p. 640-3.

${ }^{4}$ GARCÍA, LERA-LÓPEZ, y SUÁREZ. Estimation of a structural model of the determinants of the time spent on physical activity and sport: Evidence for Spain, p. 519-21.
} 
El objetivo de este trabajo es analizar la vinculación de diferentes variables socioeconómicas (edad, género, edad, educación, hogar, ingresos, ocupación y hábitos deportivos) con la práctica del running en Argentina. Nuestro propósito de aportar conocimiento empírico para la elaboración de estrategias que impulsen a las personas a adoptar y mantener la práctica del running como actividad física recurrente.

\section{Metodología}

Dados los objetivos que nos planteamos, adoptamos para nuestro trabajo un enfoque cuantitativo que apela a fuentes secundarias. En la realización de este trabajo recurrimos a la base de datos de la Encuesta Nacional de Factores de Riesgo (ENFR) de 2009, realizada en conjunto por el INDEC, el Ministerio de Salud y la Secretaría de Deportes. La ENFR 2009 incluyó -hasta ahora por única vez- una Encuesta Nacional de Actividad Física y Deportiva (ENAFyD), con preguntas específicas referidas a la práctica de la actividad física y deportiva de cada individuo encuestado.

El diseño muestral de la ENFR 2009 fue probabilístico, estratificado por conglomerados y multietápico. La selección del encuestado se hizo aleatoriamente aplicando la tabla de selección de Kish y las entrevistas a las personas encuestadas fueron presenciales y personales (autoinformante). Los valores muestrales se expandieron aplicando factores de expansión correspondientes a cada etapa de selección, con correcciones por no respuesta y calibración de pesos iniciales. La encuesta consta de tres bloques: vivienda, hogar e individual. Incluye una muestra efectiva de 34.732 personas mayores de 18 años, que viven en ciudades de más de 5.000 habitantes en todo el país. Además de los datos sociodemográficos y de salud de la ENFR se recolectaron datos acerca de las actividades físicas y deportivas practicadas o no en los últimos 30 días previos a la entrevista. Se indagó acerca del tipo de actividad física o deportiva de preferencia (practicada y/o deseada), lugar y frecuencia de práctica y los motivos de práctica o de no práctica. La base de datos de esta encuesta fue publicada por el INDEC en 2018.

Para analizar la vinculación de diferentes variables socioeconómicas con las categorías de análisis recurrimos a regresiones logísticas y análisis de 
correspondencias múltiples. En el procesamiento de los datos utilizamos el paquete de software estadístico IBM SPSS Statistics Versión 20.

\section{CATEgorizaCión DEL UNIVERSO DE ESTUDIO}

En este trabajo denominamos running a la práctica no profesional, en mayor o menor medida sostenida en el tiempo, de lo que en la ENAFyD se denomina "Correr". Los tiempos o ritmos de carrera abarcan un espectro amplio, ${ }^{5}$ y salvo para algunos pocos practicantes, son relevantes sólo para cada corredor. A tal punto que la alternancia de carrera y caminata, conocida como método Galloway, es aceptada incluso por corredores avanzados. El running es una actividad "potencialmente" muy inclusiva, en términos de género, edad, costos e infraestructura requerida. ${ }^{6}$

El universo de los corredores dista de ser uniforme y requiere categorizarse para su mejor análisis. En su estudio etnográfico de las carreras urbanas, Gil considera que "el colectivo running en Argentina está compuesto por actores con diverso grado de compromiso con la práctica y adhesión a sus preceptos éticos y estéticos", que van, como un continuo, desde un compromiso pleno y radical con la actividad hasta el abandono del estilo de vida. ${ }^{7}$ Complementando este enfoque con el de los trabajos de Smith ${ }^{8}$ y Shipway, Holloway y Jones, ${ }^{9}$ adoptamos los siguientes criterios analíticos para la categorización de nuestro universo de estudio:

1) Runners: personas que definen el correr como su actividad deportiva más importante y corren tres o más veces por semana.

2) Fun runners: personas que definen el correr como su actividad deportiva más importante y corren menos de tres veces por semana.

\footnotetext{
${ }^{5}$ A modo de ejemplo, el ritmo de carrera en la maratón de Buenos Aires de 2018 fue de 2:58 $\mathrm{min} / \mathrm{km}$ (2h $5 \mathrm{~min})$ para el ganador y 9:11 $\mathrm{min} / \mathrm{km}$ (6h $27 \mathrm{~min}$ ) para el último clasificado.

${ }^{6}$ HIJÓS. La historia del running en Argentina, p. 123. En este trabajo cuestionaremos en parte esta visión

${ }^{7}$ GIL. Correr y competir. Rituales de interacción y estilo de vida en el running, p. 109.

${ }^{8}$ SMITH. Athletes, runners, and joggers: participant-group dynamics in a sport of "individuals", $p$. 189.

${ }^{9}$ SHIPWAY, HOLLOWAY, y JONES. Organizations, practices, actors, and events: Exploring inside the distance running social world, p. 263.
} 
3) Deportistas que corren: personas que practican otros deportes y corren, pero no consideran el correr como su actividad deportiva más importante (corren como complemento).

\section{¿NACIDOS PARA CORRER?}

A pesar predisposición genética del ser humano para las carreras de larga distancia ${ }^{10}$ y a las olas de crecimiento que llevaron al running a convertirse en una actividad de masas, ${ }^{11}$ existen variables económicas, individuales y sociales que condicionan su adopción. ${ }^{12}$ En ese mismo sentido, Eren ${ }^{13}$ destaca que los corredores de fondo tienden a ser hombres adultos, de clase media, educados y con empleos bien pagos en tareas profesionales. En este trabajo nos proponemos analizar cómo se vinculan diferentes variables socioeconómicas con nuestras categorías de estudio en Argentina, incluyendo la comparación con otras actividades deportivas en el mapa perceptual.

Análisis de regresión de los factores que afectan a la participación en el running

Si bien relaciones entre las categorías analizadas y distintas variables socioeconómicas podrían analizarse mediante tablas bivariadas sencillas, consideramos que esta técnica no captura apropiadamente la interrelación entre variables. Para ello es más adecuado recurrir a modelos de regresión, que permiten examinar a las variables en conjunto, como un todo, teniendo en cuenta interacciones tales como "el servicio doméstico en Argentina está conformado en gran medida por mujeres" o "los patrones y directivos tienden a tener ingresos más altos y educación superior". En este trabajo desarrollamos modelos de regresión logística binaria, que vinculan a la participación en las diferentes categorías

\footnotetext{
${ }^{10}$ MATTSON. Evolutionary aspects of human exercise—born to run purposefully, p. 348.

${ }^{11}$ SCHEERDER, BREEDVELD, y BORGERS. Running across Europe: the rise and size of one of the largest sport markets, p. 12.

${ }^{12}$ GARCIA, LERA-LÓPEZ, y SUÁREZ. Estimation of a structural model of the determinants of the time spent on physical activity and sport: Evidence for Spain, p. 519-521.

${ }^{13}$ EREN. Estranged Labor, Habitus, and Verstehen in the Rise of Extreme Endurance Sports, p. 3.
} 
analizadas con los distintos factores sociodemográficos. ${ }^{14}$ Para ello realizamos modelos individuales para las categorías Runner, Fun runner y Deportista que corre, e incorporamos también un modelo ("Corredores") que consolida a todas las categorías anteriores. Para el cálculo de los modelos utilizamos la función Regresión logística binaria de SPSS, que tiene la forma:

$$
\ln \left(\frac{p}{1-p}\right)=\beta X+\varepsilon
$$

donde $p$ es la probabilidad de participación y $\beta$ y $X$ son vectores con 29 elementos (incluyendo la constante). Todas las variables explicativas del modelo son binarias (dummy) y representan a los factores género, edad, educación, tipo de hogar, ingreso del hogar (en quintiles), estatus ocupacional, tiempo trabajado y práctica de otros deportes. La ventaja de usar modelos de regresión logística binaria en lugar de modelos de regresión de cuadrados mínimos es que los resultados generados por los modelos logísticos están diseñados para cubrir un rango de resultados desde un mínimo de cero hasta un máximo de uno. Esto los hace ideales para trabajar con variables dependientes binarias - por ejemplo, la participación en una categoría de running - donde cero representa la no participación y uno la participación. Los resultados de los modelos de cuadrados mínimos pueden caer fuera del dominio cero-uno, lo que los hace menos apropiados para este tipo de variables. Los modelos de regresión logística binaria son no lineales y no asumen variaciones constantes en los resultados de la misma forma en que lo hacen los coeficientes de los modelos lineales. Por eso reportamos los efectos de las variables como cambios en la participación esperada comparada con la categoría de referencia a partir de la comparación del valor de sus coeficientes $(B)$ o de su razón de probabilidades (odds ratio) $\operatorname{Exp}(B)$.

Para calcular la expectativa de participación en cada modelo tenemos que resolver la ecuación de regresión en términos de $p$. En el caso de la categoría de referencia:

${ }^{14}$ PENG; SO. Logistic regression analysis and reporting: A primer. p. 31-70. 


$$
\ln \left(\frac{p}{1-p}\right)=c
$$

donde $c$ es la constante, entonces resolviendo para $p$ :

$$
\operatorname{Exp}(c)=\frac{p}{1-p} \Rightarrow p=\frac{\operatorname{Exp}(c)}{1+\operatorname{Exp}(c)}
$$

En el modelo de la categoría Runner, c es igual a -2,52, $\operatorname{Exp}(c)$ es igual a 0,08 y p es igual a 7,4\%. Para comparar el efecto de ser "mujer" en contraposición a ser "hombre" ceteris paribus, en lugar de comparar entre ambos atributos en las estadísticas descriptivas, comparamos los atributos Género: Mujer; Edad: 18-24 años; Educación: Superior; Tipo de hogar: Unipersonal; Ingreso del hogar: Quintil 5 (superior); Estatus ocupacional: Patrones y directivos profesionales; Tiempo trabajado:<35 horas semanales; con los atributos Género: Hombre; Edad: 18-24 años; Educación: Superior; Tipo de hogar: Unipersonal; Ingreso del hogar: Quintil 5 (superior); Estatus ocupacional: Patrones y directivos profesionales; Tiempo trabajado: $<35$ horas semanales. Para calcular la nueva probabilidad esperada $(p)$ tenemos que:

$$
\operatorname{Exp}(c+B)=\frac{p^{\prime}}{1-p^{\prime}} \Rightarrow p^{\prime}=\frac{\operatorname{Exp}(c+B)}{1+\operatorname{Exp}(c+B)}=\frac{\operatorname{Exp}(c) \times \operatorname{Exp}(B)}{1+\operatorname{Exp}(c) \times \operatorname{Exp}(B)}
$$

donde $B$, para el atributo "mujer" en el modelo de la categoría Runner, es -0,72 y $p^{\prime}$ es igual a 3,8\%. Si bien $p^{\prime}-p(7,4 \%-3,8 \%)$ compara el efecto de ser "mujer" con el de ser "hombre" manteniendo constantes el resto de los atributos de la categoría de referencia, el cambio de magnitud de $p$ a $p^{\prime}$ depende del valor de $p$. Es decir que no existe una relación uno a uno entre el valor de un coeficiente y su efecto en la probabilidad de participación, por lo que la definición de la categoría de referencia es importante para definir la posición en la que trabajaremos en la curva logística. La categoría de referencia en los modelos construidos corresponde al siguiente perfil sociodemográfico: 1. Género: Hombre; 2. Edad: 18-24 años; Educación: Superior; Tipo de hogar: Unipersonal; Ingreso del hogar: Quintil 5 (superior); 
Estatus ocupacional: Patrones y directivos; Tiempo trabajado: $<35$ horas semanales; Practica otros deportes: Sí. Seleccionamos esta categoría de referencia tomando los atributos que, de acuerdo con la bibliografía y nuestros descriptivos estadísticos, hacen que la probabilidad de practicar el running sea máxima, por lo que la mayoría de los atributos deberían tener efecto negativo en la probabilidad de participación. En nuestras tablas exponemos los coeficientes $B$ y sus razones de probabilidad $\operatorname{Exp}(B)$, que tienen los siguientes efectos en la probabilidad de participación:

- Cuando $B<0, \operatorname{Exp}(B)>0 \mathrm{y}<1$, el efecto del atributo es negativo

- Cuando $B \approx 1, \operatorname{Exp}(B) \approx 1$, el efecto del atributo es neutro

- Cuando $B>1, \operatorname{Exp}(B)>1$, el efecto del atributo es positivo

La intensidad del efecto es mayor cuanto más alejado esté $\operatorname{Exp}(B)$ de 1, es decir que un $\operatorname{Exp}(B)$ igual a 0,08 indica un efecto más negativo que un $\operatorname{Exp}(B)$ igual a 0,48.

Resultados: La Tabla 1 detalla las variables utilizadas en los modelos, su categorización y frecuencia ponderada, basados en un tamaño de muestra probabilística 34.366 casos (sin expandir), equivalentes a una muestra ponderada de 24,2 millones de casos.

\begin{tabular}{|c|c|c|c|c|c|}
\hline \multirow[b]{2}{*}{ Características individuales } & $\begin{array}{c}\text { Frecuencia } \\
\text { sin ponderar }\end{array}$ & Porcentaje & \multirow{3}{*}{$\begin{array}{l}\text { Características eonómicas } \\
\text { Ingreso del hogar }\end{array}$} & \multirow[t]{2}{*}{$\begin{array}{c}\text { Frecuencia } \\
\text { sin ponderar }\end{array}$} & \multirow[t]{2}{*}{ Porcentaje } \\
\hline & & & & & \\
\hline Género & & & & & \\
\hline Hombre & 14836 & $43,2 \%$ & Quintil 5 (superior) & 6175 & $18,0 \%$ \\
\hline \multirow[t]{2}{*}{ Mujer } & 19530 & $56,8 \%$ & Quintil 4 & 6237 & $18,1 \%$ \\
\hline & & & Quintil 3 & 6258 & $18,2 \%$ \\
\hline Edad & & & Quintil 2 & 6988 & $20,3 \%$ \\
\hline $18-24$ años & 4649 & $13,5 \%$ & Quintil 1 (inferior) & 8708 & $25,3 \%$ \\
\hline 25-34 años & 7549 & $22,0 \%$ & & & \\
\hline $35-49$ años & 9463 & $27,5 \%$ & Estatus ocupacional & & \\
\hline 50-64 años & 6984 & $20,3 \%$ & Patrones y directivos profesionales & 744 & $2,2 \%$ \\
\hline \multirow[t]{2}{*}{ 65-más años } & 5721 & $16,6 \%$ & Patrones y directivos no profesionales & 1720 & $5,0 \%$ \\
\hline & & & Cuenta propia calificados & 2473 & $7,2 \%$ \\
\hline Características sociales & & & Asalariados profesionales & 2436 & $7,1 \%$ \\
\hline Educación (completa o incompleta) & & & As alariados no profesionales & 6268 & $18,2 \%$ \\
\hline Superior & 8851 & $25,8 \%$ & Asalariados informales & 3339 & $9,7 \%$ \\
\hline Media & 13252 & $38,6 \%$ & Cuenta propia no calificados & 2715 & $7,9 \%$ \\
\hline \multirow[t]{2}{*}{ Básica } & 12263 & $35,7 \%$ & Servicio doméstico y trab. familiares & 1652 & $4,8 \%$ \\
\hline & & & Desocupado & 1422 & $4,1 \%$ \\
\hline Tipo de hogar & & & Inactivo & 11597 & $33,7 \%$ \\
\hline Unipersonal & 5358 & $15,6 \%$ & & & \\
\hline Conyugal sin hijos ni otros & 4309 & $12,5 \%$ & \multicolumn{2}{|c|}{ Tiempo trabajado (excluye Desocupados e Inactivos) } & \\
\hline Conyugal con hijos & 13896 & $40,4 \%$ & $<35$ hs semanales & 7501 & $35,1 \%$ \\
\hline Conyugal con hijos y otros & 2799 & $8,1 \%$ & 35 a 45 hs semanales & 8279 & $38,8 \%$ \\
\hline Cony, incompleto con hijos y/u otros & 6463 & $18,8 \%$ & $>45$ hs semanales & 5567 & $26,1 \%$ \\
\hline \multirow[t]{5}{*}{ No conyugal múltiple } & 1541 & $4,5 \%$ & & & \\
\hline & & & \multicolumn{2}{|l|}{ Características deportivas } & \\
\hline & & & \multicolumn{2}{|l|}{ Practica otro deporte } & \\
\hline & & & $\mathrm{Si}$ & 13014 & $37,9 \%$ \\
\hline & & & No & 21352 & $62,1 \%$ \\
\hline
\end{tabular}

Tabla 1 - Número de casos $(\mathrm{N})$ de la muestra sin expandir por atributo analizado. $\mathrm{N}$ (sin expandir) = 34366 casos, equivalentes a 24,2 millones de casos ponderados. Fuente: Elaboración propia con datos de la ENFR 2009. 
La Tabla 2 resume los resultados de los modelos de regresión de cada categoría analizada. Todas las variables y atributos de los modelos resultaron significativas con un nivel de confianza del 99\% (Sig. <0,01). En el proceso de estructuración de los modelos eliminamos algunas de las variables analizadas -por ejemplo, Estado civil, Cantidad de menores de 18 años en el hogar y Cantidad de miembros del hogar, entre otras-. Las razones de probabilidad de las constantes en cada modelo son: Runner: 0,08; Fun runner: 0,08; Deportista que corre: 0,34; Corredores: 0,70. En líneas generales los modelos de las categorías de corredores tienen aspectos similares, en cuanto a que todos muestran una mayor probabilidad de participación de hombres, con educación superior, ingresos altos y que practican otros deportes.

De cara a validar internamente nuestros modelos y garantizar la solidez de la muestra y la distribución de categorías, recurrimos a la técnica de remuestreo bootstrapping con 100 iteraciones, a partir de los que determinamos los intervalos de confianza al 95\% de los coeficientes B y su significación. Con excepción de algunos coeficientes de la categoría Estatus ocupacional, los coeficientes B de los modelos son tienen significancia al nivel de 0,01.

De acuerdo con nuestros datos, el ser mujer reduce la probabilidad de participar en cualquiera de las categorías de corredores a menos de la mitad que el ser hombre (Runner: $\operatorname{Exp}(B)=0,49 ;$ Fun runner: $\operatorname{Exp}(B)=0,41$ y Deportista que corre: $\operatorname{Exp}(B)=0,33)$.

La edad tiene un impacto negativo en las probabilidades de practicar el running, pero con diferencias entre categorías. Mientras que la probabilidad de participación en la categoría Runner es máxima a en el rango de 35-49 años $(\operatorname{Exp}(B)=1,25)$ para luego caer hasta $\operatorname{Exp}(B)=0,10$ en el rango de 65 años y más, las categoría Fun runner y Deportista que corre muestran $\operatorname{Exp}(B)$ decrecientes partiendo de la categoría de referencia.

El running se vincula en todas sus categorías con la educación superior (completa o incompleta). El no alcanzar este nivel educativo representa una reducción sustancial en la probabilidad de participación, con $\operatorname{Exp}(B)$ entre 0,55 y 0,61. La caída en las probabilidades se acentúa si el nivel educativo alcanzado es sólo el básico.

El tipo de hogar no muestra un patrón claro de participación en las categorías vinculadas al running, aunque, en general, los hogares unipersonales y no 
conyugales múltiples tienen $\operatorname{Exp}(B)$ mayores. Los hogares unipersonales son los de menor $\operatorname{Exp}(B)$ en la categoría Inactivo no vinculado. Un hallazgo interesante es la baja probabilidad de participación en la categoría Runner en hogares conyugales sin hijos ni otros, con $\operatorname{Exp}(B)=0,55$ (el mínimo de esta variable en la categoría).

Los ingresos del hogar tienen una relación positiva pero no lineal con la práctica del running. La caída en $\operatorname{los} \operatorname{Exp}(B)$ de cada quintil varía por categoría, siendo los quintiles 5 y 4 los de mayor probabilidad de participación en todos los casos. El valor máximo de $\operatorname{Exp}(B)$ de la categoría Runner se da en el quintil $4(1,12)$.

En cuanto al estatus ocupacional, no encontramos en nuestros datos patrones claros de comportamiento, al punto de categorías de baja tasa de participación muestran $\operatorname{Exp}(B)$ inesperadamente altos. Por ejemplo, la probabilidad de participación en la categoría Fun runner es máxima $-\operatorname{Exp}(B)=1,56$ - para Servicio doméstico y trabajadores familiares. Entendemos que esto se debe a que este grupo está conformado en un 90\% por mujeres, en un 39\% por hogares del quintil 1 de ingresos y en un $41 \%$ por individuos con educación básica, factores todos que hacen a una baja probabilidad de participación en la categoría. Aparece como distintivo la mayor probabilidad de participación de Patrones y directivos profesionales y no profesionales en la categoría Runner.

El tiempo trabajado se vincula negativamente con todas las categorías vinculadas al running, que muestran $\operatorname{Exp}(B)$ máximos en menos de 35 horas trabajadas por semana. Excluyendo la variable Estatus ocupacional para eliminar redundancias, puede apreciarse que la probabilidad de participación en el running de individuos desocupados es comparativamente alta -Runner: $\operatorname{Exp}(B)=1,12$; Fun runner: $\operatorname{Exp}(B)=1,23 ;$ y Deportista que correr: $\operatorname{Exp}(B)=0,96)-$.

La participación en el running tiene un fuerte vínculo positivo con la práctica simultánea de otros deportes. La probabilidad de que un deportista pertenezca a la categoría Runner triplica a la de un no deportista $-\operatorname{Exp}(B)=0,32$ para quienes no practican otros deportes-, mientras que para las categorías de running en su conjunto la probabilidad de los deportistas es ocho veces mayor que la de los no deportistas. 
FuLiA/UFMG - [...] variables socioeconómicas en la práctica del running en Argentina

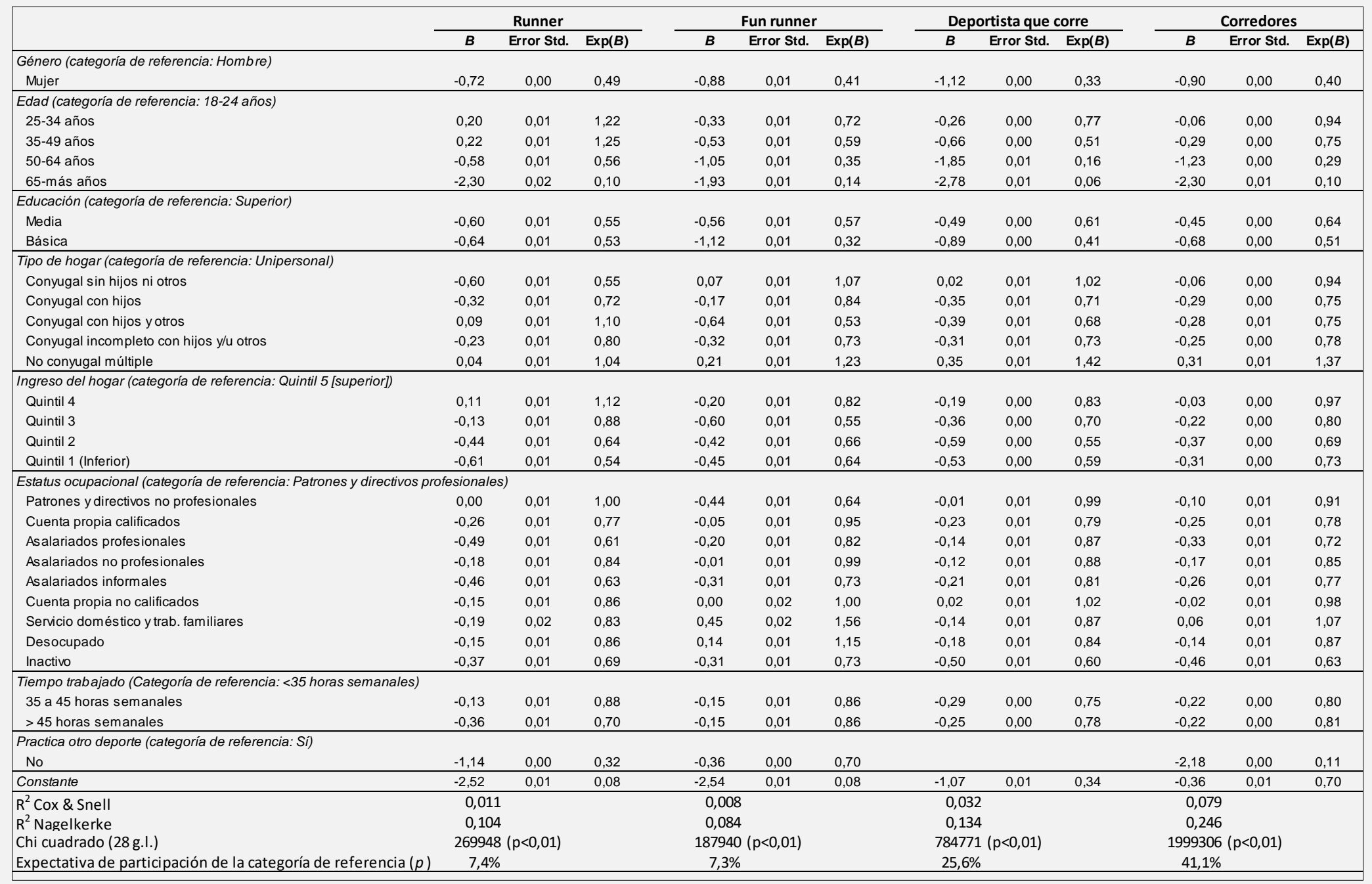

Tabla 2 - Modelos de participación por categoría de corredores. Fuente: Elaboración propia con datos de la ENFR 2009. 
Análisis de correspondencias múltiples (ACM) de las actividades físicas y deportivas

Si bien nuestro tema de investigación es el running, el estudio de cualquier deporte en particular requiere tener en cuenta el conjunto de las demás prácticas deportivas, con el propósito de comprender las afinidades entre ciertos deportes y ciertas características de los agentes ubicados en determinada posición del espacio social. ${ }^{15}$ Para ello decidimos emplear el análisis de correspondencias múltiples (ACM), una técnica multivariada factorial de reducción de dimensiones que se utiliza para el análisis exploratorio de datos multidimensionales. El uso de esta técnica ayuda a descubrir interrelaciones entre las características medidas, poniéndolas de manifiesto en gráficos bidimensionales (mapas perceptuales) que pueden ser interpretados con relativa facilidad. Debido a su carácter descriptivo y exploratorio, la técnica de ACM no requiere hipótesis ni supuestos de ninguna naturaleza.

Para nuestro ACM incorporamos algunas de las variables con las que trabajamos anteriormente: Género, Edad, Educación, Tipo de hogar, Ingreso del hogar (quintiles) y Estatus ocupacional. Sumamos la variable Deporte practicado y quitamos la variable Tiempo trabajado (para evitar redundancias que distorsionaban el análisis). La reducción de dimensiones muestra un eje-dimensión 1- asociado las variables Estatus ocupacional, Educación e Ingresos del hogar (Inercia=0,33), y un segundo eje -dimensión 2- asociado a las variables Edad y Estatus ocupacional (Inercia $=0,29$ ) la Figura 1 muestra las medidas de discriminación con la relación de cada variable con las dimensiones 1 y 2 .

Debido a que el software estadístico que utilizamos ${ }^{16}$ no dispone de una herramienta para aplicar la técnica de bootstrapping al ACM, hicimos un ejercicio de remuestreo manual para validar internamente nuestros análisis y garantizar la solidez de los clusters identificados. Tomamos tres submuestras, cada una con $30 \%$ de los casos totales seleccionados al azar, con las que repetimos nuestro ACM. Los tres mapas perceptuales mantuvieron la misma estructura de distribución de puntos que el mapa general, aunque con rotaciones en los ejes.

${ }^{15}$ GÓMEZ ROJAS, GRINSZPUN, y SEID. Clases de deporte y deportes de clase: La distribución de los gustos y prácticas deportivas en el espacio social, p. 8.

${ }^{16}$ IBM SPSS Statistics Versión 20. 
El gráfico bidimensional de las variables categóricas analizadas en el plano de las dimensiones 1 y 2 (Figura 2) muestra el espacio social del deporte en relación con dichas variables. Con el propósito de identificar diferentes clusters, segmentamos el plano de las dimensiones 1 y 2 con una grilla ortogonal con la misma pendiente que el vector de discriminación de Deporte practicado. Cabe aclarar que esta forma de segmentar el plano no se corresponde con ninguna técnica reconocida, sino que fue la seleccionada entre varias opciones trabajadas en el proceso de encontrar agrupaciones con sentido y valor teórico.

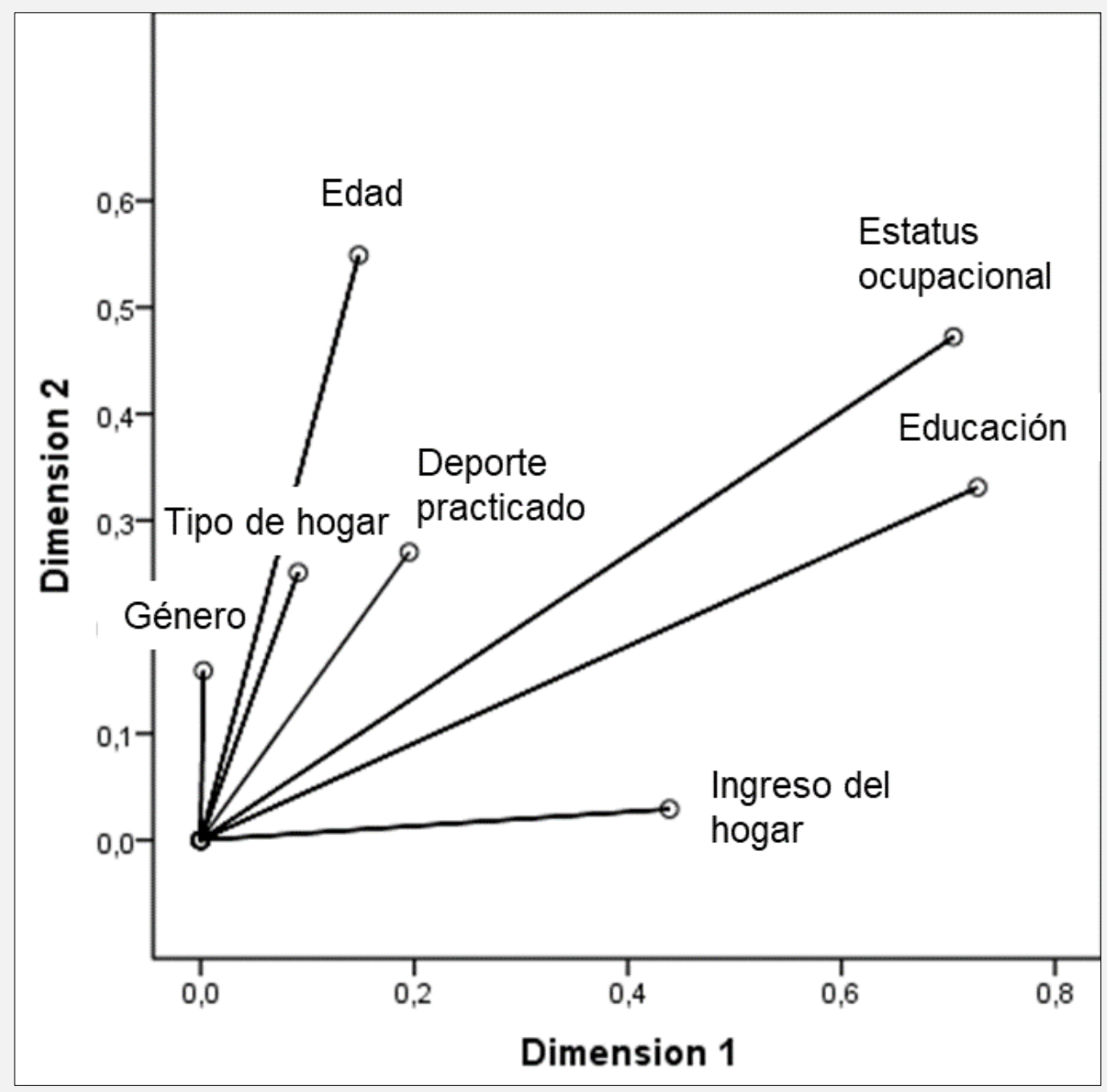

Figura 1 - Medidas de discriminación.

Fuente: Elaboración propia con datos de la ENFR 2009. 


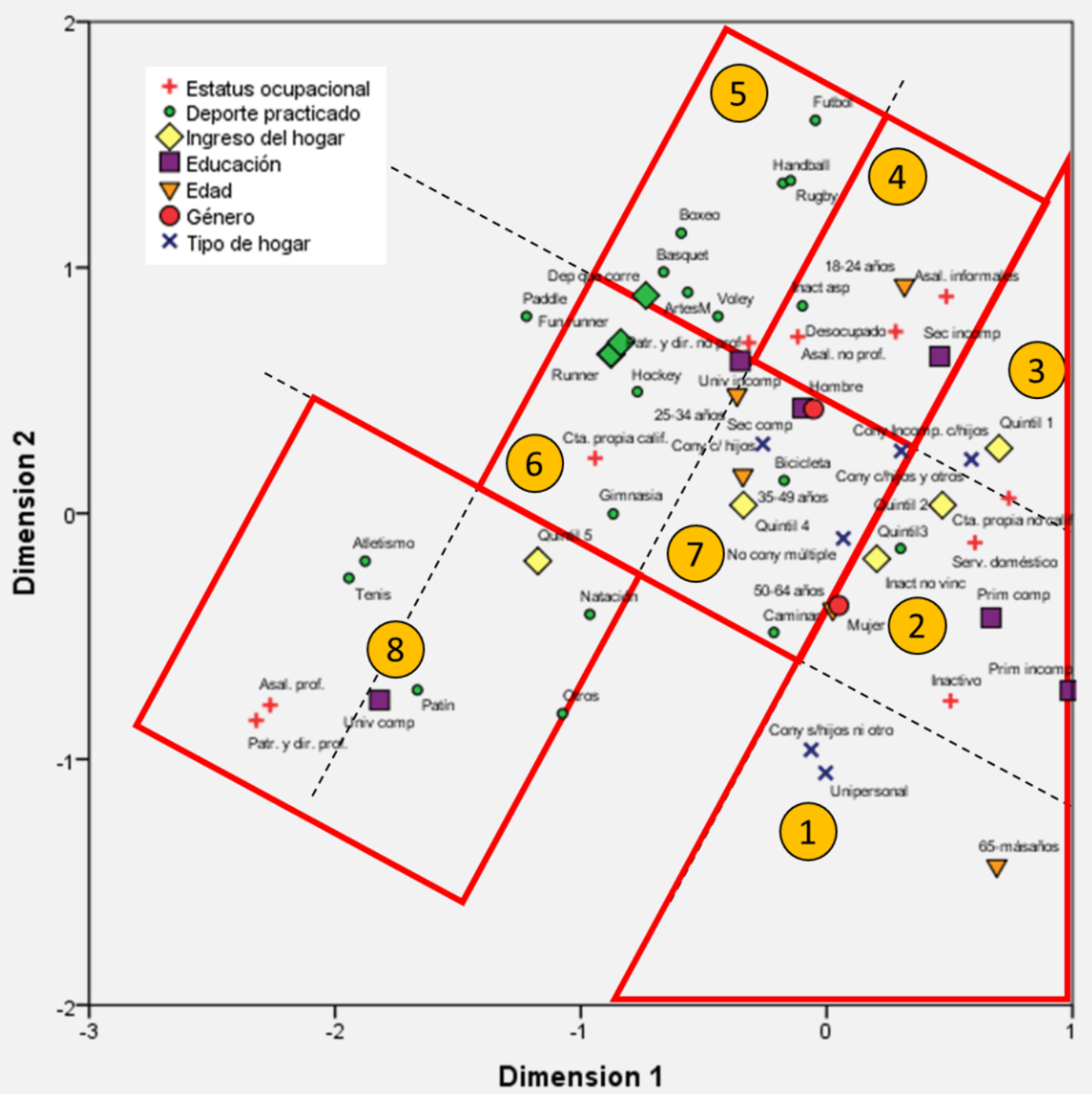

Figura 2 - Diagrama conjunto de puntos de categoría. Fuente: Elaboración propia con datos de la ENFR 2009.

Esta grilla nos permite en primera instancia identificar un segmento o cluster alejado de la práctica deportiva, al que dividimos a su vez en tres subsegmentos con las siguientes características: 1) Individuos de 65 años o más e inactivos en lo referente a su estatus ocupacional; 2) Mujeres, individuos con educación básica, empleados de servicio doméstico y cuentapropistas no calificados, de ingresos medios y medios-bajos (Q2 y Q3); 3) Hombres jóvenes de ingresos bajos (Q1).

Moviéndonos hacia arriba y a la izquierda, tenemos el segmento 4) que siendo aún inactivo manifiesta interés por correr. Está conformado por hombres jóvenes con educación secundaria incompleta, con ingresos medios y medios-bajos y con baja calificación ocupacional. 
Continuando el movimiento hacia arriba y a la izquierda, pasamos al segmento 5) con hombres jóvenes, de ingresos medios altos (Q4) y estudios universitarios incompletos. Este segmento incluye a la mayor parte de los deportes "populares" -como el fútbol, el rugby y el básquet, entre otros-, asociados al despliegue físico en los que el cuerpo tiene un valor instrumental.

Descendiendo al centro de nuestro mapa perceptual, encontramos un segmento, subdividido a su vez en los subsegmentos 6) y 7), conformados por adultos de 25 a 49 años, de ingresos altos y medio-altos (Q5 y Q4) con estudios universitarios incompletos y buena calificación ocupacional. En líneas generales, los deportes de este segmento -correr, ciclismo o gimnasia- están vinculados con lo que Bourdieu llamó "disposiciones ascéticas de individuos en ascensión", 17 en las que el cuerpo no es ya un instrumento sino un fin en sí mismo. Se trata de deportes que son practicados por el mantenimiento físico y el beneficio social que proporcionan. El subsegmento 6) corresponde a individuos relativamente más jóvenes y de mayores ingresos y nivel educativo que los del subsegmento 7). Las categorías Runner y Fun runner pertenecen al subsegmento 6). Cabe destacar que, pese a las diferencias identificadas anteriormente entre ambas categorías, en nuestro mapa perceptual se encuentran muy próximas entre sí. El subsegmento 7) incluye -además del ciclismo- el caminar, aunque en el borde geométrico del subsegmento, próximo a los individuos de 50 a 64 años, en lo que podría presumirse como una evolución de la actividad deportiva dentro segmento con el paso de los años.

Finalmente, el extremo inferior izquierdo tenemos al segmento 8), conformado por individuos de ingresos altos, alta calificación ocupacional y estudios universitarios completos. Las actividades deportivas en este segmento -por ejemplo el tenis- requieren aprendizaje, están estetizadas y se corresponden con ámbitos de exclusividad.

Dejamos a la cercanía del paddle y el hockey con el running como anomalías del análisis para las que no encontramos explicación con los datos disponibles.

\footnotetext{
17 BOURDIEU. La distinción. Criterios y bases sociales del gusto, p. 211.
} 


\section{DiSCUSIÓN Y CONCLUSIONES}

En este trabajo intentamos evaluar cuantitativamente la relación de diferentes variables sociodemográficas con la práctica del running en sus diferentes categorías analíticas, la interacción entre dichas variables en sus efectos sobre la participación en cada categoría analítica y la posición del running en un mapa perceptual del mundo social del deporte. En líneas generales, nuestros análisis permiten sostener que las características individuales, sociales y económicas -por ejemplo, la edad, el género, la educación, los ingresos y los hábitos deportivos- tienen un efecto relevante sobre la participación en el running. Es decir que la práctica del running está condicionada al mismo tiempo por lo biológico y lo social. Nuestros estudios cuantitativos coinciden con los hallazgos etnográficos de Hijós, ${ }^{18}$ quien sostiene que “[...] a pesar de que el running es un deporte 'abierto' a todas las clases sociales, existen límites económicos y culturales en el acceso total a la práctica”, y con los de Gil, ${ }^{19}$ quien señala que muchos de los aficionados al running gozan de un elevado capital social y pueden montar redes de colaboración para ayudar económicamente a algunos atletas. Coincidentemente con nuestro mapa perceptual, Acciaresi halló en su etnografía de un running team de La Plata que casi todos los miembros del grupo pertenecían un estrato social medio/medio alto, destacadamente profesionales, empleados formales, profesores, estudiantes universitarios y cuentapropistas. ${ }^{20}$

El running es una actividad comparativamente masculina, ya que la probabilidad de participación de un hombre duplica a la de una mujer. Si bien históricamente la diferencia en participación se atribuyó a aspectos biológicos -entre otros, las diferencias físicas o la maternidad-, el crecimiento en la participación femenina en distinto tipo de carreras de fondo en la Argentina y el mundo apunta a una discriminación por género de carácter eminentemente social, que se traduce en una alta participación de la inactividad física entre las mujeres. La amplia participación de mujeres en los running teams, destacadas en las etnografías

\footnotetext{
18 HIJÓS. "¿Todos podemos ser corredores?”: Un análisis sobre la comunidad runner y sus vínculos con el mercado, p. 33.

${ }^{19}$ GIL. Correr y competir. Rituales de interacción y estilo de vida en el running, p. 110.

${ }^{20}$ ACCIARESI. "Ya se me había hecho un hobby, una droga": goce, dolor e identificación en un grupo platense de corredores, p. 31.
} 
de Acciaresi e Hijós, permitiría inferir que la participación femenina en el running continuará aumentando. En esta línea, Garton e Hijós consideran que "la creciente participación de las mujeres en las competencias de resistencia se traduce como un habilitación y legitimidad en la práctica deportiva para el sexo femenino".21

La edad del individuo es un factor de alta relevancia en la práctica del deporte en general y también del running. La probabilidad de practicar la actividad se reduce con la edad, especialmente a partir de los 50 años. Cabe destacar, sin embargo, que la categoría Runner muestra probabilidades de participación máximas en el rango de 35 a 49 años, en lo que podría ser una manifestación de la superadherencia de los corredores identificada por Glasser. ${ }^{22}$ Acciaresi destaca que "Las edades [de los corredores] son de lo más diversas: hay personas de hasta casi 60 años, con años de entrenamiento, una mayoría de personas entre 30 y 45 años, y un pequeño grupo menor a 30 años de edad". Cabe preguntarse si pasado el umbral de los 50 años los corredores pasan a ser inactivos o no. En este sentido, Acciaresi identifica la importancia que le asigna un corredor de 53 años "al hecho de sentirse bien, de estar bien 'por dentro' y, en ese sentido, tener la sensación de tener menor edad de la que tiene". ${ }^{23}$ Nuestra segmentación del mapa perceptual llevaría a pensar que algunos podrían volcarse a prácticas menos exigentes desde lo físico, típicamente caminar. Por su parte, la inactividad física asociada a la edad es uno de los clusters que identificamos en nuestro mapa perceptual. Si bien a priori puede asociarse a la edad como un condicionante biológico, Koronios, Mavromati, Kriemadis, et al. ${ }^{24}$ señalan aspectos sociales vinculados a la menor actividad deportiva de los adultos mayores, como los estilos de vida, la falta de información acerca de los beneficios del deporte o la falta de confianza en la propia capacidad corporal.

La probabilidad de practicar el running ( $\mathrm{y}$ en general cualquier deporte) aumenta con el nivel educativo, especialmente cuando se accede a estudios superiores, aunque sean incompletos. El acceso a la educación superior duplica la

\footnotetext{
${ }^{21}$ GARTON; HIJÓS. "La deportista moderna": Género, clase y consumo en el fútbol, running, y jockey argentinos, p.15.

${ }^{22}$ GLASSER. Promoting client strength through positive addiction, p. 174.

${ }^{23}$ ACCIARESI. "Ya se me había hecho un hobby, una droga": goce, dolor e identificación en un grupo platense de corredores, p. 10, 71 .

${ }^{24}$ KORONIOS, MAVROMATI, KRIEMADIS, et al. Sport participation and ageing: Evidence from marathon events, p. 130.
} 
probabilidad de práctica del running en cualquiera de sus categorías. Nuestros modelos de regresión muestran que el efecto positivo de la educación en la probabilidad de correr excede al efecto de lo puramente económico, con el que está correlacionado. Es posible que la educación formal brinde la posibilidad de acceder a distintas experiencias deportivas y una mejor comprensión de los beneficios del ejercicio. Ese capital cultural podría devenir en un mayor capital corporal en una relación recursiva. ${ }^{25}$ En mayor nivel educativo incrementa también la probabilidad de que la población inactiva manifieste interés por correr, especialmente en los estadios iniciales (el paso de educación básica a educación media duplica la probabilidad de estar interesado). Consecuentemente, la combinación de inactividad física con bajo nivel educativo es otro de los clusters identificados en nuestro mapa perceptual. Estos hallazgos refuerzan los planteos de Hijós respecto de los límites culturales que limitan el acceso a la práctica del running. ${ }^{26}$

El estado civil y el tipo de hogar (tanto en su conformación como en la cantidad de miembros) parecen tener un vínculo indirecto con el running, vinculado posiblemente con el tiempo disponible para dedicar a la actividad. El no tener responsabilidades familiares, en tanto que ser soltero o no tener hijos, parece tener un efecto positivo en la actividad. Se trata, de todas formas, de una variable de impacto comparativamente menor y sin un patrón claro.

Desde el punto de vista de las variables económicas, el running muestra una vinculación con los quintiles de mayores ingresos ( 4 y 5). Si bien no es una actividad asociada a la elite -como el tenis-, dista de tener el alcance popular que tienen otras actividades como el fútbol. A pesar de que la probabilidad máxima de participación en el running se da en Patrones y directivos, nuestro mapa perceptual lo agrupa con la categoría de Cuentapropistas calificados, es decir que se trata de una actividad de individuos con buen nivel de ingresos y formación pero que no pertenecen a una elite profesional. La disponibilidad de tiempo es una variable relevante que apareció indirectamente cuando analizamos el efecto del Tipo de hogar. El dedicar más horas al trabajo, para un nivel de ingresos determinado, afecta negativamente la

\footnotetext{
${ }^{25}$ WACQUANT. Un arma sagrada. Los boxeadores profesionales: capital y trabajo corporal, p. 242. ${ }^{26}$ HIJÓS. "¿Todos podemos ser corredores?": Un análisis sobre la comunidad runner y sus vínculos con el mercado, p. 33.
} 
probabilidad de practicar el running. La afectación es mayor para la categoría Runner que para la categoría Fun runner, lo que a priori es razonable dado que el tiempo semanal dedicado a correr es mayor en la primera que en la segunda. Estos hallazgos coinciden con los de los trabajos etnográficos citados anteriormente.

En cuanto al efecto de los hábitos deportivos, la probabilidad de que un deportista practique el running (como primera opción o como complemento) es ocho veces mayor que la de alguien sin un deporte alternativo. Esto puede explicarse tanto desde una perspectiva individual -por transferencia de habilidades, agilidad, capacidad aeróbica y muscular, coordinación, etc.-, como desde una perspectiva social, que señala que las disposiciones hacia el deporte constituyen una dimensión de la relación con el propio cuerpo y se inscriben en un estilo de vida. A partir de ambas perspectivas, puede deducirse que el paso más crítico para estimular la práctica del running (y cualquier otro deporte) es el de salto de la inactividad a la actividad deportiva en cualquiera de sus formas.

El running comparte un espacio relativamente cercano a los sectores más favorecidos de la sociedad y alejado por lo tanto de los sectores más desfavorecidos. Retomando el propósito de nuestro trabajo, el diseño de estrategias de promoción del running como forma de estimular su práctica recurrente debe tener en cuenta que esta disciplina no tiene aún el alcance suficiente para llegar a todos estratos de la sociedad. Dados algunos patrones comunes entre el running y caminar, las "correcaminatas" o las distancias menores participativas (no competitivas) podrían ser la forma de convertir las maratones en fiestas populares, en el sentido más amplio del término.

Finalmente nos gustaría mencionar que, estando este trabajo en revisión (junio de 2021), el Ministerio de Turismo y Deportes de la Nación anunció el lanzamiento en 2021 de la segunda Encuesta Nacional de Actividad Física y Deportiva. Consideramos que podría ser de interés replicar este trabajo con los resultados de la nueva encuesta para evaluar el impacto en el running de las restricciones a la movilidad implementadas en Argentina durante 2020 a raíz de la pandemia de COVID-19. 


\section{REFERENCIAS}

ACCIARESI, Pablo. "Ya se me había hecho un hobby, una droga": Goce, dolor e identificación en un grupo platense de corredores. Tesis de grado. Universidad Nacional de La Plata. FaHCE, 2014.

BOURDIEU, Pierre.La distinción. Criterios y bases sociales del gusto. Madr id: Taurus, 1998.

DOWNWARD, Paul. Exploring the economic choice to participate in sport: Results from the 2002 General Household Survey. International review of applied economics, v. 21 n. 5, p. 633-53, 2007.

EREN, Colleen. Estranged Labor, Habitus, and Verstehen in the Rise of Extreme Endurance Sports. Journal of Sport and Social Issues, v. 41 n. 5, p. 1-18, 2017.

GARCÍA, Jaume; LERA-LÓPEZ, Fernando; y SUÁREZ, María José. Estimation of a structural model of the determinants of the time spent on physical activity and sport: Evidence for Spain. Journal of Sports Economics, v. 12, n. 5, p. 51537, 2011.

GARTON, Gabriela; e HIJÓS, Nemesia. "La deportista moderna”: género, clase y consumo en el fútbol, running, y jockey argentinos. Antípoda, revista de Antropología y Arqueología, v. 30, p. 23-42, 2018.

GIL, Gastón. Correr y competir. Rituales de interacción y estilo de vida en el running. Revista del Museo de Antropología, v. 12, n. 1, p. 105-16, 2019.

GLASSER, William. Promoting client strength through positive addiction. Canadian Journal of Counselling and Psychotherapy, v. 11, n. 4, 1977.

GÓMEZ ROJAS, Gabriela; GRINSZPUN, Marcela; y SEID, Gonzalo. Clases de deporte y deportes de clase: La distribución de los gustos y prácticas deportivas en el espacio social. En VII Jornadas de Sociología de la UNLP: "Argentina en el escenario latinoamericano actual: Debates desde las ciencias sociales". Universidad Nacional de La Plata. Facultad de Humanidades y Ciencias de la Educación. Departamento de Sociología, 2012.

HIJÓS, Nemesia. La historia del running en Argentina. Materiales para la Historia del Deporte, v. 17, p. 122-35, 2018.

HIJÓS, Nemesia. “¿Todos podemos ser corredores?”: Un análisis sobre la comunidad runner y su vínculo con el mercado. Arquivos em movimento, v. 14, p. 22-38, 2018.

KORONIOS, Konstantinos; MAVROMATI, Marina; KRIEMADIS, Athanasios; LEIVADITI, Eleni; KOLOVOS, Petros; y PAPADOPOULOS, Andreas. Sport participation and ageing: Evidence from marathon events. In: GeNeDis. Springer, Cham, 2017, p. 129-39.

MATTSON, Mark. Evolutionary aspects of human exercise-born to run purposefully. Ageing research reviews, v. 11 n. 3, p. 347-52, 2012.

PENG, Chao-Ying; SO, Tak-Shing. Logistic regression analysis and reporting: A primer. Understanding Statistics: Statistical Issues in Psychology, Education, and the Social Sciences, v. 1, n. 1, p. 31-70, 2002. 
SCHEERDER, Jeroen; BREEDVELD, Koen; y BORGERS, Julie. Eds.. Running across Europe: the rise and size of one of the largest sport markets. Palgrave Macmillan, London, 2015.

SHIPWAY, Richard; HOLLOWAY, Immy; y JONES lan. "Organizations, practices, actors, and events: Exploring inside the distance running social world", International Review for the Sociology of Sport, v. 48 n. 3, p. 259-76, 2013.

SMITH, Stuart. Athletes, runners, and joggers: participant-group dynamics in a sport of "individuals". Sociology of Sport Journal, v. 15 n. 2, p. 174-92, 1998

WACQUANT, Loïc. Un arma sagrada. Los boxeadores profesionales: capital corporal y trabajo corporal. En: J. AUYERO. Caja de herramientas - El lugar de la cultura en la sociología norteamericana. Buenos Aires: Unqui, 1999, p. 237-92.

Recebido para publicação em: 23 mar. 2021. Aprovado em: 15 jun. 2021. 